\title{
Gramicidin Ion Channel-Based Biosensors: Construction, Stochastic Dynamical Models, and Statistical Detection Algorithms
}

\author{
Vikram Krishnamurthy, Fellow, IEEE, Kai Yiu Luk, Bruce Cornell, Jog Prashar, Isabelle L. di Maio, \\ Hedayetul Islam, Andrew R. Battle, Stella M. Valenzuela, and Donald K. Martin
}

\begin{abstract}
This paper deals with the experimental construction, stochastic modeling, and statistical signal processing of a novel, artificially constructed biosensor comprised of biological ion channels. Such nanoscale biosensors have been built by incorporating dimeric gramicidin A (bis-gA) ion channels into bilayer membranes of giant unilamellar liposomes, and then excising small patches of the membrane loaded with ion channels. We present a stochastic model for the response of the biosensor and present statistical model validation tests to verify the adequacy of the model. We show that in the presence of specific target molecules, the statistics of the gating mechanisms of the $\mathrm{gA}$ channels are altered. By capturing the change in real time, we devise a maximum-likelihood detector to detect the presence of target molecules. To test the sensitivity of this model, we conducted patch-clamp experiments with two compounds known to inhibit conduction of the gA channels. We found experimentally that the real-time detection algorithm was able to accurately identify the addition of the compounds even when the alterations in the patch-clamp recordings were very small. This algorithm provides the sensitive detection system for ongoing development of lipid-based nanosensors.
\end{abstract}

Index Terms-Biosensor, estimation, gramicidin A, lipid membrane, maximum-likelihood detection.

\section{INTRODUCTION}

B IOLOGICAL ion channels are protein molecules commonly found in cell membranes that form water filled nanotubes, typically a few Angstrom units in radius. In biological systems, ion channels selectively regulate the flow

Manuscript received January 2, 2007; revised February 27, 2007; accepted March 5, 2007. The work of D. K. Martin, S. M. Valenzuela, and B. Cornell was supported in part by the Australian Research Council under Grant LP0560749. The work of D. K. Martin was supported in part the Backing Australia's Ability Program of the Department of Education Science and Training under Grant CG060027 that supported I. L. di Maio, H. Islam, and A. R. Battle with Postdoctoral Fellowships. The associate editor coordinating the review of this paper and approving it for publication was Prof. Ooi Kiang.

V. Krishnamurthy and K. Y. Luk are with the Department of Electrical and Computer Engineering, University of British Columbia, Vancouver, BC V6T 1Z4, Canada (e-mail: vikramk@ece.ubc.ca; kluk@ece.ubc.ca).

B. Cornell is with Surgical Diagnostics Pty Ltd., St. Leonards, NSW 2065, Australia (e-mail: brucec@surgicaldiagnostics.com).

J. Prashar is with Ambri Pty Ltd., Chatswood, NSW 2063, Australia (e-mail: jogp@ambri.com).

I. L. di Maio, H. Islam, A. R. Battle, S. M. Valenzuela, and D. K. Martin are with the Department of Medical and Molecular Biosciences, University of Technology, Sydney, NSW 2007, Australia (e-mail: isabelle.di-maio@orange.fr;Hedayetul.Islam@uts.edu.au; a.battle@uq.edu.au; Stella.Valenzuela@uts.edu.au; donm@uts.edu.au).

Color versions of one or more of the figures in this paper are available online at http://ieeexplore.ieee.org.

Digital Object Identifier 10.1109/JSEN.2007.901254 of ions into and out of a cell and, thus, regulate the cell's electrical activity. By exploiting the selective conductivity of ion channels in the presence of target molecules, biosensors have been developed to detect molecular species of interest across a wide range of applications. These include medical diagnostics, environmental monitoring, and general biohazard detection. In particular, a novel biosenor, which incorporated monomeric gramicidin $\mathrm{A}$ ion channels into a tethered lipid bilayer membrane and exploited the changes in the association and disassociation probabilities of the Gramicidin dimers, was published by a coauthor of this paper in Nature [1]. For a detailed NMR analysis of the monomeric gA channels, please refer to [2].

This paper deals with the construction, modeling, and statistical signal processing associated with a new type of ion channel based biosensor.

1) Construction of Biosensor: The biosensor constructed in this paper comprises engineered dimeric gramicidin A ion channels incorporated into a lipid bilayer membrane, supported over a $1-\mu \mathrm{m}$ diameter opening of a micropipette, which was excised from a giant lipid vesicle. In a giant lipid vesicle, covalent dimeric gramicidin $\mathrm{A}$ ion channels were incorporated by codispersion with the vesicle forming lipids. This type of artificially constructed biosensor mimics the naturally occurring ion transport processes of a living cell.

2) Stochastic Dynamical Models for Biosensor: Having constructed the biosensor, we formulated a stochastic dynamical model to capture its experimental behavior. The engineered dimeric gA channels provided the conducting pore for the vesicle membrane, but not with the normal kinetics of simple monomeric $\mathrm{gA}$ that normally diffuses in the inner and outer leaflets of a bilayer membrane. The gating mechanism of the dimeric $\mathrm{gA}$ ion channels in the biosensor is thought to arise from the random movement of excess lipid lenses in the liposome that diffuse over the membrane surface and block the conducting channels. In experiments, we found that a hidden Markov model (HMM) [3], which takes into account of the $1 / \mathrm{f}$ noise in the biosensor's response, is an adequate model for the biosensor currents.

3) Statistical Detection Algorithms for Target Molecules: In the presence of target molecules, the stochastic behavior of the biosensor current changes. By using a sequential maximum-likelihood detector, we show that the biosensor can be used in real-time target molecule detection. We illustrate the use of the biosensor in detecting two types 

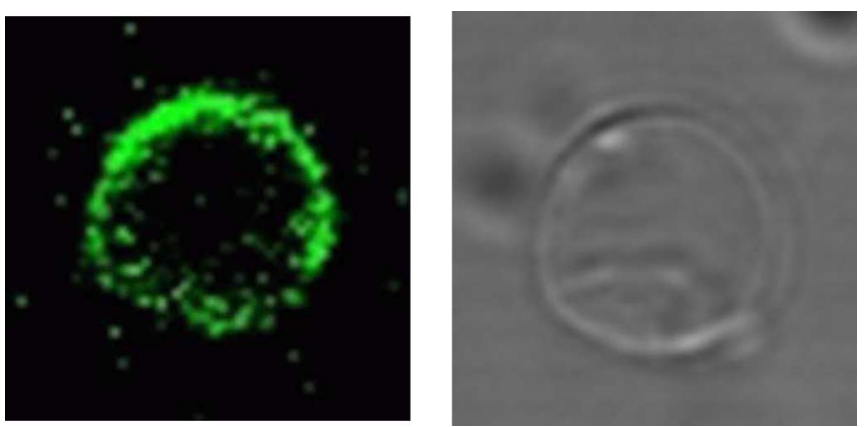

Fig. 1. Fluorescence image (left) of biosensor's horizontal optical section shows the bis-gA channels labeled using FITC and identified by the green color. Phase-contrast image (right) of the same horizontal slice shows the overall shape of the biosensor.

of target molecules, methylbenzthonium chloride (MBC) and 2-methyl-4-tert.-octylphenol (MTOP). The experimental data presented shows that the detection algorithm performed remarkably well even when it was difficult to visually identify the model change. Thus, the system we present in this paper provides a sensitive platform for the development of artificially constructed biosensors that better mimic the function of living cells.

The remainder of this paper is organized as follows. Section II describes the construction of the biosensor. Section III presents a HMM to describe the dynamical behavior of the biosensor. We also present model validation methods to verify the goodness-of-fit of the HMM to the biosensor response and sequential detection algorithms for detecting target molecules in real-time; and finally, in Section IV, we demonstrate the experimental detection of two target moecules, MBC and MTOP. Both compounds are known to inhibit the conduction of bis-gA channels.

\section{EXPERIMENTAL CONSTRUCTION OF THE bis-gA Model BIOMIMETIC ION CHANNEL BIOSENSOR}

The biosensor considered in this paper was constructed by incorporating bis-gA ion channels into the lipid bilayer membrane of giant unilamellar liposomes,, and then excising small patches $(1 \mu \mathrm{m}$ in diameter) of the lipid membrane using a patch-clamp micropipette. The bis-gA was synthesized at the Ambri laboratories. Fig. 1 shows the fluorescence and phase-contrast image of the optical section through the diameter of the biosensor. The solutions and chemicals used for the model biosensor was purchased from Sigma Chemical Company (St. Louis, MO) and included DL-alpha-phosphatidylcholine (PC) from soybean, cholesterol, chloroform, sucrose, glucose, sodium chloride, potassium chloride, and 4-(2-Hydroxyethyl)piperazine-1-ethanesulfonic acid (HEPES). Giant unilamellar liposomes were prepared using our recent protocol [4] that was modified from a standard hydration procedure [5]-[7]. In brief, a dried lipid film was prepared from $100 \mu \mathrm{L}$ of $10 \mathrm{mg} \cdot \mathrm{mL}^{-1}$ PC with $10 \%(\mathrm{w} / \mathrm{w})$ or $40 \%(\mathrm{w} / \mathrm{w})$ cholesterol in chloroform in a glass test tube, as described earlier. Bis-gA (1:100 mole/mole) was added and rehydration of the lipid was made at $45^{\circ} \mathrm{C}$ by the addition of a small amount of pure water $(5 \mu \mathrm{L})$ to the tube for a few minutes followed by the addition of $5 \mathrm{~mL}$ of an aqueous solution of $0.1 \mathrm{M}$ or $0.2 \mathrm{M}$ sucrose. The tube was incubated at $45^{\circ} \mathrm{C}$ for $2-3 \mathrm{~h}$.

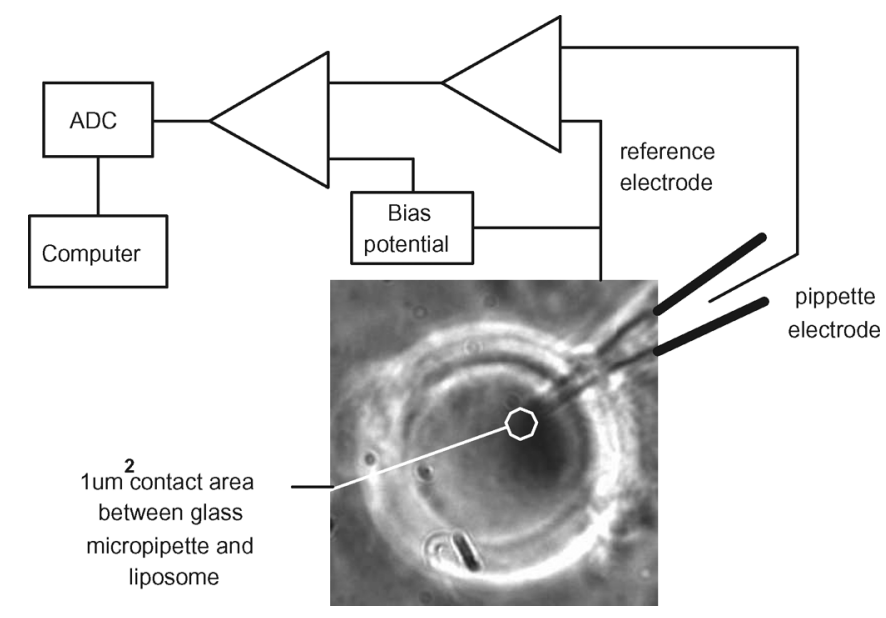

Fig. 2. Photo of glass micropipette and liposome with block diagram of the experimental setup.

After gentle rocking overnight at room temperature, the lipid film dispersed uniformly and a white cloud was floating in the middle of the solution, which contained giant liposomes. The obtained liposomes were approximately $20 \mu \mathrm{m}$ in diameter and were stable up to four days at $4{ }^{\circ} \mathrm{C}$.

Patch-clamp electrophysiology was used to record the ionic currents from the model biosensor that resulted from the permeation of ions through the bis-gA ion channels incorporated in the lipid membrane patches that were excised from the giant unilamellar liposomes. To perform the patch-clamp recordings, the liposomes were allowed to settle on the bottom of a recording chamber, which was either a 35-mm plastic tissue-culture dish or a purpose-built recording bath fitted with the glass cover-slip (Warner, RC13). Patch-clamp pipettes with a tip opening between 0.9 and $1.5 \mu \mathrm{m}$ were fabricated from borosilicate capillary glass tubing (Modulohm A/S, Denmark, Vitrex 1601) using an automated puller (Sutter, P97). The patch-clamp pipette was used to excise patches of lipid membrane from the giant unilamellar liposomes. The bis-gA channel currents from these membrane patches were amplified and filtered at $1 \mathrm{kHz}$ (four-pole Bessel) using an Axopatch 200B amplifier (Axon Instruments) and sampled online at $10 \mathrm{kHz}$. Fig. 2 shows a schematic of the experimental setup.

\section{Stochastic Modeling and Statistical Model} VALIDATION OF BIOSENSOR RESPONSE to TARGET MOLECULES

In this section, we discuss estimation techniques to extract the model parameters of the biosensor response. Statistical model validation tests are introduced to verify the goodness-of-fit of the model. With the biosensor response fitted to the dynamical model, we present the maximum-likelihood detection algorithm for detecting target molecules.

\section{A. Stochastic Modeling of Biosensor}

Suppose a patch clamp experiment is conducted with $(N-1)$ gramicidin A channels in the biosensor. At each discrete time instant $k$, each bis-gA channel can be either in the "open" or "closed" state and each open channel conducts a fixed current. Thus, the total current due to all $(N-1)$ ion channels at any given time can take on one of $N$ possible levels $\left\{\mu_{1}, \ldots, \mu_{N}\right\}$ 


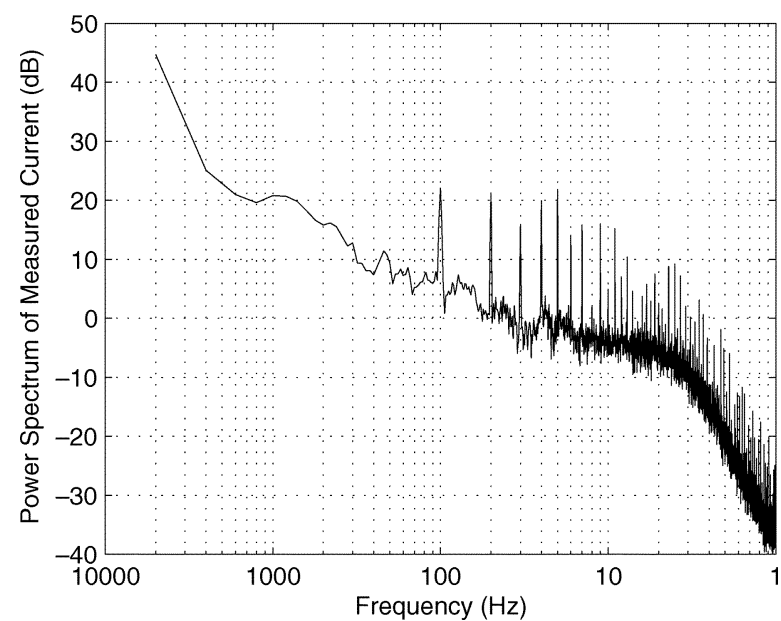

Fig. 3. Power spectral density of biosensor response clearly shows the $1 / \mathrm{f}$ open channel noise and the anti-aliasing effect in the samples.

and can be modeled as a $N$-state Markov chain. Write as $\mu=$ $\left(\mu_{1}, \ldots, \mu_{N}\right)$. Let $X_{k}$ denote the total channel current at discrete time $k$. Let

$$
a_{i j}=P\left(X_{k}=\mu_{j} \mid X_{k-1}=\mu_{i}\right), \quad i, j \in\{1, \ldots, N\}
$$

denote the transition probabilities of the Markov chain. Also, let

$$
\pi_{0}(i)=P\left(X_{1}=\mu_{i}\right), \quad i \in\{1, \ldots, N\}
$$

denote the initial distribution of the Markov chain. Write $A=$ $\left[a_{i j}\right]_{N \times N}$ and $\pi_{0}=\left[\pi_{0}(i)\right]_{N \times 1}$. The measured current from the biosensor is a distorted version of the signal $X_{k}$. The distortion arises from thermal noise, the anti-aliasing effect from sampling and an open channel noise with its power proportional to the inverse of frequency. Thus, this is also known as $1 / \mathrm{f}$ noise and is discussed in other studies of bis-gA ion channels [8], [9]. Fig. 3 shows the power spectral density of a typical sequence of biosensor recordings and clearly shows that the power decreases at a rate of $-10 \mathrm{~dB} / \mathrm{dec}$ at low frequencies and has a sharp cutoff at approximately $1 \mathrm{kHz}$. To model this correlated noise process, we can use an auto-regressive (AR) Gaussian process that comprises white Gaussian noise process $W_{k}$ filtered by an all-pole filter. We represent the filter with transfer function $H\left(q^{-1}\right)$, where $q^{-1}$ denotes the unit delay operator

$$
Y_{k}=X_{k}+\frac{W_{k}}{H\left(q^{-1}\right)}
$$

or equivalently

$$
H\left(q^{-1}\right) Y_{k}=H\left(q^{-1}\right) X_{k}+W_{k}
$$

where

$$
H\left(q^{-1}\right)=1+h_{1} q^{-1}+\ldots+h_{M} q^{-M} .
$$

However, the 1/f noise is a long memory process and requires choosing the filter order $M$ to be large. The state space of $H\left(q^{-1}\right) X_{k}$ in (3) increases exponentially with $M$, thus making it computationally infeasible to model long memory processes like $1 / \mathrm{f}$ noise. We observe that in our current setup, the gating mechanism of the bis-gA channels is a much slower process relative to the sampling rate and most of the power of the stochastic process resides at DC. Therefore, we argue that we can closely approximate the behavior of the gramicidin channels with our model in (4), provided that the filter $H(z)$ has unity DC gain. We redefine the response of the noisy observation as

$$
Y_{k}=\frac{X_{k}+W_{k}}{H\left(q^{-1}\right)}
$$

We show with model validation methods in Section III-B that the model in (4) accurately describes the biosensor response. Write $\mathbf{h}=\left(1, h_{1}, \ldots, h_{M}\right)^{T}$. It is convenient to model the noise corrupting the state of the biosensor as state dependent noise - that is the noise variance at any given time instant is dependent on the state of the biosensor at that time instant. Let $\sigma_{i}^{2}$ be the variance of state $i, i=(1, \ldots, N)$. Write $\sigma^{2}=$ $\left(\sigma_{1}^{2}, \ldots, \sigma_{N}^{2}\right)$. As a result, the observations can be formulated as a HMM sequence. Let $\theta=\left(A, \pi, \mu, \sigma^{2}, \mathbf{h}\right)$ be the HMM that characterizes the output measured current from the biosensor.

The use of stochastic modeling as part of a biosensing platform eliminates the majority of electrical and mechanical interfering effects. The approach further offers the ability to enhance the specificity and affinity of biosensor through the use of receptor combinations in conjunction with analysis by stochastic modeling.

\section{B. Maximum-Likelihood Estimation (MLE) of Biosensor Parameters}

Given an observation sequence $\left\{Y_{k}\right\}$ of length $T$, we define $L_{k}(\theta)$ as the log-likelihood of our model at discrete time $k$. The estimation of the model $\theta$ involves processing $\left\{Y_{k}\right\}$ through a HMM maximum-likelihood estimator (MLE). The system in (4) can be rewritten as

$$
\mathbf{h}^{T} \mathbf{Y}_{k}=X_{k}+W_{k}
$$

where $\mathbf{Y}_{\mathbf{k}}=\left(Y_{k}, Y_{k-1}, \ldots, Y_{k-M}\right)^{T}$. This formulation is analogous to a standard HMM, except that the observation sequence is filtered by a finite-impulse response (FIR) filter. The expectation maximization (EM) algorithm is an iterative procedure that solves for local maximum of the likelihood function. The E-step evaluates the log-likelihood, which is defined as

$$
\begin{aligned}
L_{k}(\theta)=\sum_{t=1}^{k} \sum_{i=1}^{N} \gamma_{t}(i) \log ( & \left.\frac{1}{\sqrt{2 \pi \sigma_{i}^{2}}} \exp \left(\frac{-\left(\mathbf{h}^{T} \mathbf{Y}_{\mathbf{k}}-\mu_{i}\right)^{2}}{2 \sigma_{i}^{2}}\right)\right) \\
& +\sum_{t=1}^{k} \sum_{i=1}^{N} \sum_{j=1}^{N} \zeta_{t}(i, j) \log \left(a_{i j}\right)
\end{aligned}
$$


and the M-step maximizes the log-likelihood with respect to the model parameters as follows:

$$
\begin{aligned}
a_{i j} & =\frac{\sum_{k=1}^{T} \zeta_{k}(i, j)}{\sum_{k=1}^{T} \gamma_{k}(i)} \\
\sigma_{i}^{2} & =\frac{\sum_{k=1}^{T} \gamma_{k}(i)\left(\mathbf{h}^{T} \mathbf{Y}_{\mathbf{k}}-\mu_{i}\right)^{2}}{\sum_{k=1}^{T} \gamma_{k}(i)} \\
\mu_{i} & =\frac{\sum_{k=1}^{T} \gamma_{k}(i) \mathbf{h}^{T} \mathbf{Y}_{\mathbf{k}}}{\sum_{k=1}^{T} \gamma_{k}(i)}
\end{aligned}
$$

The coefficients of the filter $H\left(q^{-1}\right)$ can also be estimated by taking first-order derivatives of the likelihood function. Alternatively, the estimation can be implemented with the Yule-Walker type equations. For details about HMM estimation and the EM algorithm, please see [10]-[13].

\section{Statistical Model Validation of Biosensor Model}

Statistical model validation is of key importance for the biosensor since, once a satisfactory stochastic model is determined, an appropriate molecular detection algorithm can be constructed. The MLE estimates the most likely model for a fixed topology, but we need to statistically validate the adequacy of the estimated model by analyzing the autocorrelations of the residuals, which are generated via a HMM one-step predictor

$$
e_{k \mid k-1}=Y_{k}-\sum_{i=1}^{N} \sum_{j=1}^{N} a_{i j} \alpha_{k-1}(i) \mu_{j}-\sum_{n=1}^{M} h_{n} Y_{k-n}
$$

where $\alpha_{k}$ is the forward variable of the forward-backward procedure. [10] The residuals of an adequately fitted model should be uncorrelated and the autocorrelation should approach 0 as $T \rightarrow \infty$. The lag- $l$ autocorrelation function of the residual is defined as

$$
r(l)=\frac{\sum_{k=l+1}^{T}\left(e_{k}-\bar{e}\right)\left(e_{k-l}-\bar{e}\right)}{\sum_{k=1}^{T}\left(e_{k}-\bar{e}\right)^{2}}
$$

where $\bar{e}$ is the mean of the residual process. Rather than examining the autocorrelation at each lag $l$, the standard procedure is to compute the Ljung-Box Q-statistic defined in (9), which computes the cumulative sum of autocorrelations at the first $L$ lags. The Ljung-Box test is used as a portmanteau lack of fit test for model adequacy [14]

$$
Q=N(N+2) \sum_{l=1}^{L} \frac{r^{2}(l)}{(N-l)} .
$$

It is shown in [15] that for an adequate model, the Q-statistics of the residual is approximately distributed as $\chi^{2}(L)$. We will report on the validity of the model on the biosensor experimental data in Section IV.

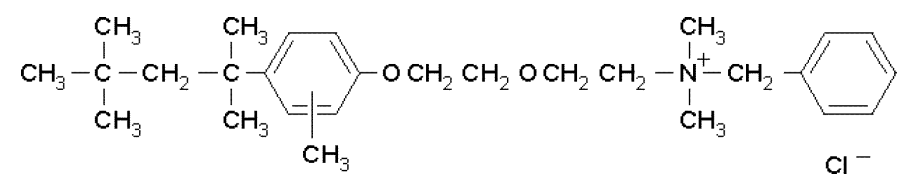

Fig. 4. Chemical structure of MBC.

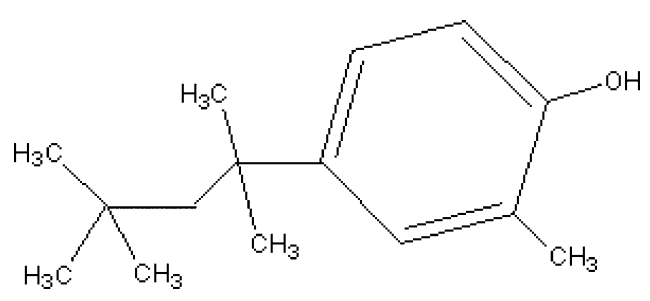

Fig. 5. Chemical structure of MTOP.

\section{Target Molecule Detection Algorithm}

After the design and validation of the dynamical models, in this section, we discuss model validation algorithms for the detection of analytes that are known to change the statistics of bis-gA channel conductance. MBC and MTOP are two analytes that are known to inhibit conductance of bis-gA channels. The chemical structure of MBC and MTOP are shown in Figs. 4 and 5, respectively. The MBC used in the experiments was purchased from Sigma Laboratories (Product Number: M7379-10G) and MTOP was prepared from MBC. The interaction of MBC and MTOP with $\mathrm{gA}$ channels is described in the manuscript [16]. The binding affinity of MBC to gA in tethered lipid bilayers and monolayers is determined to be close to $1 \mu \mathrm{m}$ in phosphate buffered saline (PBS). MTOP has a similar affinity.

Given a measured sequence observed in an unknown condition, the detection problem involves the identification of the condition that most likely contributes to the biosensor's response. This is a model classification problem and can be solved by comparing the likelihood of each known model. Let $Y=\left(Y_{1}, \ldots, Y_{T}\right)$ be a sequence of observed responses of the biosensor and let $\Theta=\left(\theta_{1}, \ldots, \theta_{M}\right)$ denote the set of model parameters that characterizes the biosensor's response for known conditions. It is assumed that at each time point $k$, the sequence $Y$ behaves according to one of $M$ possible models in $\Theta$.

The model parameters $\Theta$ are estimated and the log-likelihood at each time point $L_{k}$ can be computed from (6). To make the detection more robust to nonstationary disturbances and outliers in the measurements, we apply a geometric moving-average filter to the $\log$-likelihood. Let $\rho$ be the forgetting factor $0<\rho<1$. Define the filtered likelihood of model $\theta$ at time $k$

$$
S_{k}(\theta)=\left\{\begin{array}{ll}
L_{1}(\theta), & \text { for } k=1 \\
(1-\rho) S_{k-1}+\rho L_{k}(\theta), & \text { for } 2 \leq k \leq T
\end{array} .\right.
$$

The filtered likelihood is a weighted sum of the likelihood of the entire sequence $\left\{Y_{1}, \ldots, Y_{k}\right\}$, with higher weights on the recent observations.

The a priori probabilities of each model is generally unknown, so we devise a maximum-likelihood detector which 


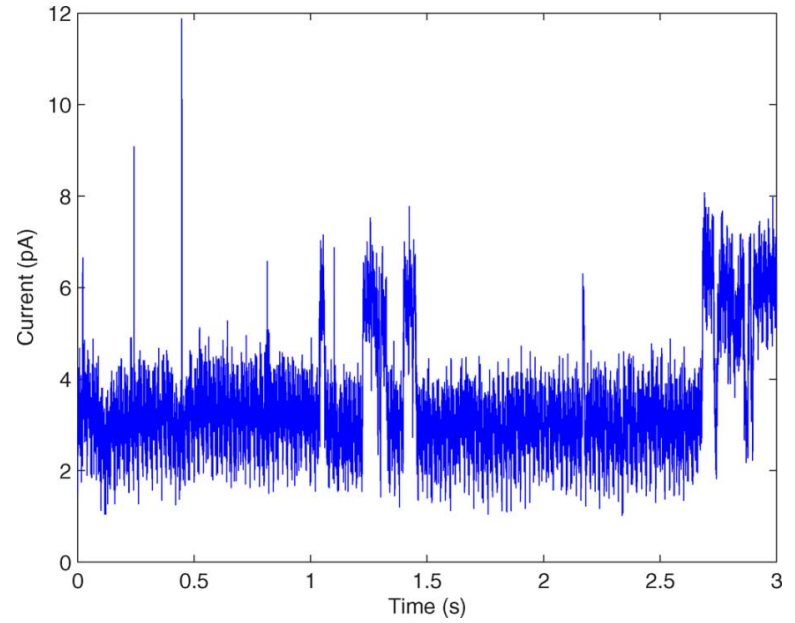

Fig. 6. Three seconds of biosensor response was recorded at an applied voltage of $50 \mathrm{mV}$ and an amplifier gain of 200 . The giant liposome was constructed from PC with $10 \%$ cholesterol in chloroform.

picks the most likely model at time $k$ given the measured sequence $Y_{1}, \ldots, Y_{k}$

$$
\hat{\theta}_{k}=\arg \max _{\theta \in \Theta}\left(S_{k}(\theta)\right)
$$

where $\hat{\theta}_{k}$ is the maximum-likelihood detection of the model at time $k$.

\section{EXPERIMENTAL RESULTS OF BIOSENSOR}

Here, we report on the goodness-of-fit of the dynamical model presented in Section III-B and the performance of the detection algorithm presented in Section III-D on actual experimental data. We recorded output from the model biosensor by measuring the activity of the bis-gA ion channels incorporated into the small lipid membrane patches that were excised from the unilamellar giant liposomes. Bis-gA ion channels were incorporated into the unilamellar giant liposomes at a concentration of $1 / 100$ from a $66 \mathrm{nM}$ stock solution. We used $0.5 \mathrm{M}$ $\mathrm{KCl}$ solution in the recording pipette with the microparticles suspended in a $0.5 \mathrm{M} \mathrm{NaCl}$ solution.

\section{A. Model Estimation and Validation for Biosensor}

We recorded the biosensor response at an applied voltage of $50 \mathrm{mV}$ and an amplifier gain of 200. Fig. 6 shows a 3-s recording of biosensor response. Notice that the bis-gA current measurements obtained here are more noisy compared to the ones obtained from a solvent sealed black lipid membrane (BLM). The quality is typical of patch clamp recordings in which a membrane patch is captured on the tip of a $1 \mu \mathrm{m}$ pipette. The seal obtained using the patch clamp technique is generally poorer than that obtained from a BLM, resulting in a worse signal-to-noise ratio for the measurements. A further potential difficulty is the possibility of multiple lipid layers being present in the patch as
TABLE I

MLE OF HMM PARAMETERS OF THE BIOSENSOR RECORDING IN FIG. 6

\begin{tabular}{|c|c|c|c|}
\hline \multicolumn{2}{|c|}{$\begin{array}{c}\text { Transition } \\
\text { Probabilities }\end{array}$} & $\begin{array}{c}\text { Conductance Level } \\
\text { (pA) }\end{array}$ & $\begin{array}{c}\text { Variance } \\
(\mathbf{p A})^{2}\end{array}$ \\
\hline 0.9964 & 0.0036 & 5.831 & 0.513 \\
\hline 0.0006 & 0.9994 & 3.057 & 0.371 \\
\hline
\end{tabular}

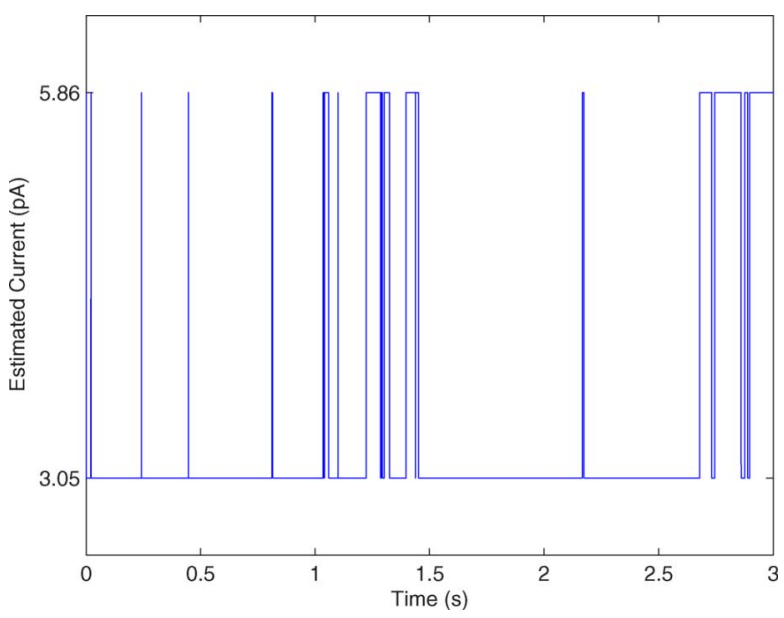

Fig. 7. Maximum-likelihood estimate of an individual conductance level was extracted from the forward-backward procedure in the HMM estimation algorithm.

compared with the BLM geometry which is thinned to a single bilayer. When recording from multiple lipid membrane stacks, the signal-to-noise ratio of the individual conduction events is reduced by noise arising from the complex impedance of the series elements.

We fitted the sequence with two-state HMM and a twelfthorder AR filter. The model parameters were estimated with the MLE and listed in Table I. The most likely conductance level sequence is extracted from the HMM procedure and is plotted in Fig. 7.

To verify that the two-state HMM outlined in Table I provides sufficient statistics to model the response of the biosensor, we computed the Q-statistics of the residuals, using (7)-(9). It can be seen in Fig. 8 that the Q-statistics are below the critical values of the chi-square distribution at 0.05 significance level for the first 13 lags. Therefore, the hypothesis that the residual is a white process cannot be rejected.

It is interesting to note that the estimated state levels, at approximately 3 and $6 \mathrm{pA}$, are rather large for a low applied voltage. One plausible explanation for this observation is that the activity of some of the bis-gA channels are correlated. In other words, the level switch from 3 to $6 \mathrm{pA}$ may be contributed by several coupled channels opening at the same time.

\section{B. Real-Time Detection of Target Molecules}

In this section, we illustrate the performance of the algorithm in detecting the presence of target molecules in real-time. We present experimental results of the biosensor in detecting two 


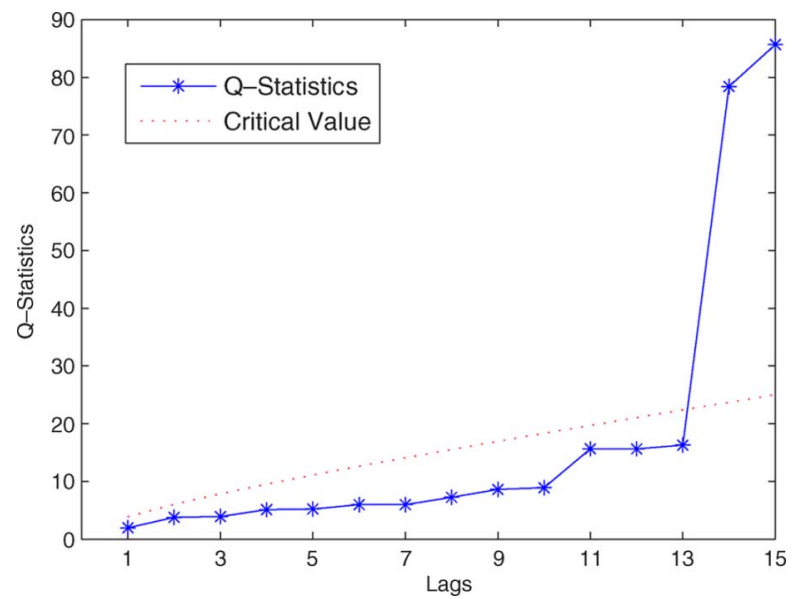

Fig. 8. For the first 13 lags, the Q-statistics of residuals are below critical value of Ljung-Box test at significance level $=0.05$. Thus, the hypothesis that the residual is a white process cannot be rejected. Therefore, the two-state HMM provides sufficient statistics to model the biosensor behavior.

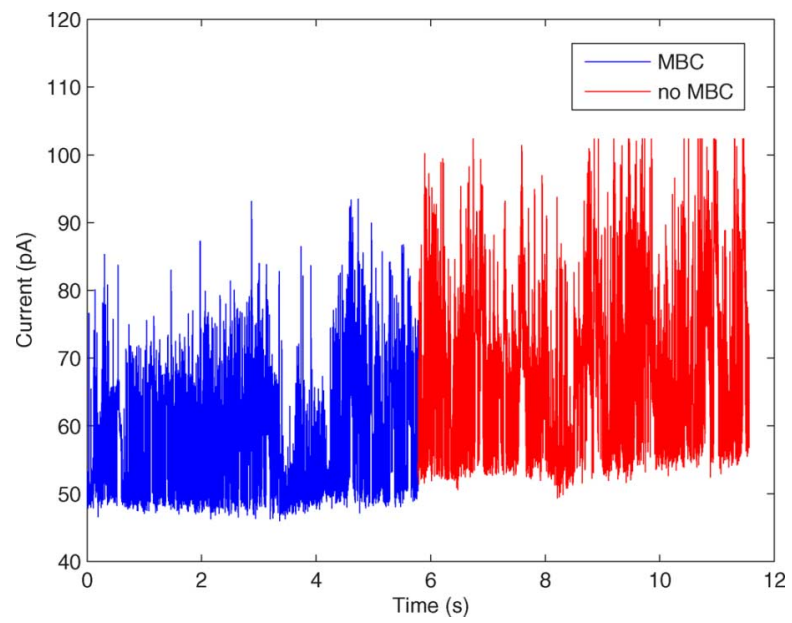

Fig. 9. The biosensor recordings with and without MBC. MBC was added after $k=57.793 \mathrm{~s}$

analytes, MBC and MTOP. Both compounds are known to inhibit conduction of the bis-gA channels. Patch-clamp experiments are conducted with and without $\mathrm{MBC}$ in the bath solution. Let

$$
\begin{aligned}
& \theta_{1}=\text { with } \mathrm{MBC} \text { in the bath solution } \\
& \theta_{2}=\text { with no } \mathrm{MBC} \text { in the bath solution. }
\end{aligned}
$$

Assuming a four-state HMM, the parameters of $\theta_{1}$ and $\theta_{2}$ are estimated with the EM algorithm. To simulate the addition of MBC into the bath solution, we merged together sequences recorded with and without $\mathrm{MBC}$. The merged sequence is plotted in Fig. 9. The filtered likelihood of each model is shown in Fig. 11. Since the stochastic model successfully captures the dependencies in the biosensor's response, the detection algorithm quickly and accurately estimates the model switching point. The detection trace in Fig. 12 indicates a switch in the

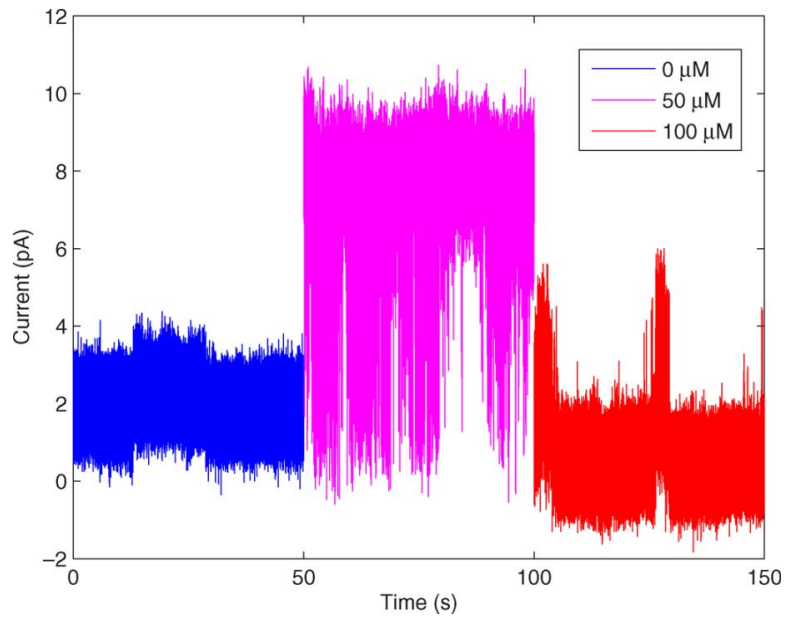

Fig. 10. The biosensor recordings with various concentrations of MTOP. $50 \mu \mathrm{M}$ of MTOP was added to the solution every $50 \mathrm{~s}$.

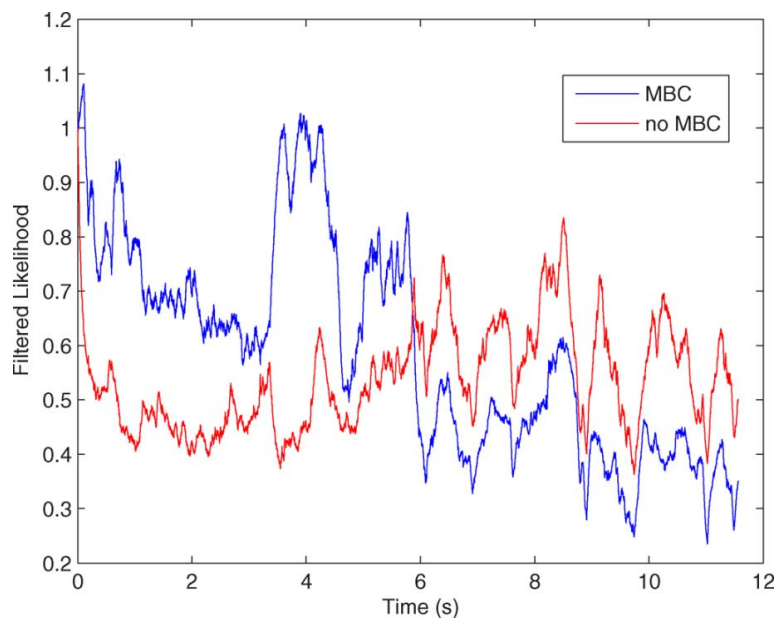

Fig. 11. The filtered likelihoods $S_{k}\left(\theta_{1}\right)$ and $S_{k}\left(\theta_{2}\right)$ computed with $\rho=0.0001$. The plot shows that the filtered likelihoods $S_{k}\left(\theta_{1}\right)$ and $S_{k}\left(\theta_{2}\right)$ were able to track the presence of MBC in the bath solution.

most likely model from $\theta_{1}$ to $\theta_{2}$ at $k=59.865 \mathrm{~s}$, approximately $0.2 \mathrm{~s}$ after MBC is added.

In the second example, we test the algorithm's performance in identifying the concentration of MTOP in the bath solution. Let

$$
\begin{aligned}
\theta_{1} & =\text { no MTOP in the bath solution } \\
\theta_{2} & =50 \mathrm{uM} \text { MTOP in the bath solution } \\
\theta_{3} & =100 \mathrm{uM} \text { MTOP in the bath solution. }
\end{aligned}
$$

The parameters of $\theta_{1}, \theta_{2}$, and $\theta_{3}$ were estimated offline with the EM algorithm. We simulated the change in concentration of MTOP by merging channel recordings independent from the training sequences. The merged sequence is plotted in Fig. 10. The filtered likelihood and the detection trace are plotted in Figs. 13 and 14, respectively. The algorithm performs extremely well in identifying the correct concentration of MTOP in the solution. 


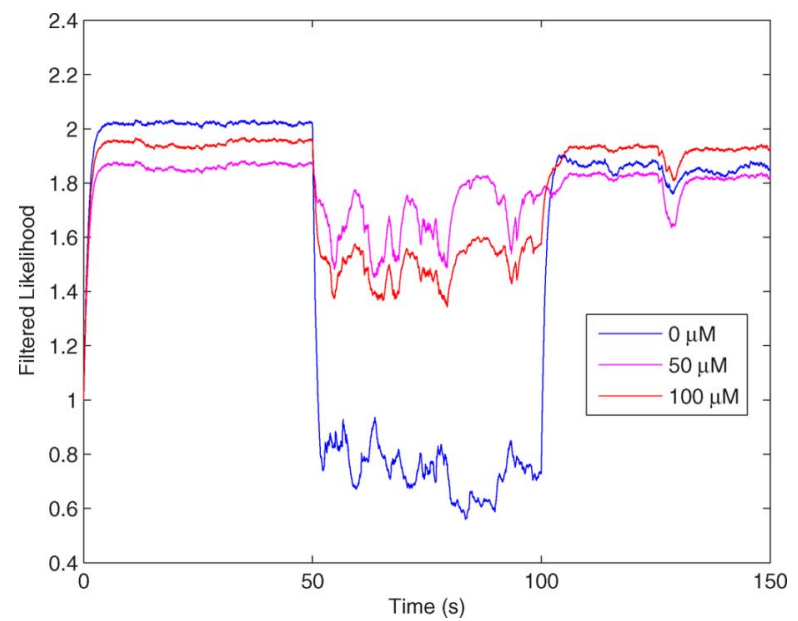

Fig. 13. The filtered likelihood $S_{k}\left(\theta_{1}\right), S_{k}\left(\theta_{2}\right)$, and $S_{k}\left(\theta_{3}\right)$ computed with $\rho=0.0001$. The plot shows that the filtered likelihoods are able to track timevarying concentrations of MTOP in the bath solution.

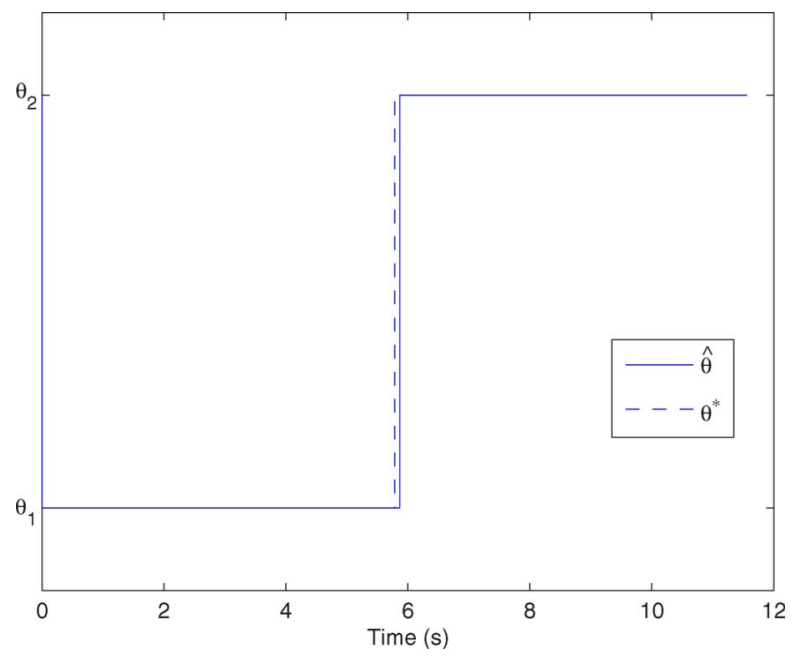

Fig. 12. Maximum-likelihood detection $\hat{\theta}$ is generated using the likelihoods in Fig. 11. While it is difficult to visually identity the transition from the recordings, the detection algorithm detected a model change shortly after MBC was added to the solution.

\section{Conclusion And Extensions}

The system we present in this paper provides a platform for the development of artificially constructed biosensors that more closely mimic the function of living cells. The biosensor comprised of engineered dimeric gramicidin A ion channels incorporated into small patches of bilayer membranes that were excised from giant unilamellar liposomes. For various experimental examples, we showed that by applying the stochastic modeling and Bayesian signal processing to detect alternations in the patch-clamp recordings, we can accurately identify additions of known analytes even when the variations are very small. This detection algorithm provides a sensitive means of detecting alterations in ion channel activity, and allows further development of this lipid-membrane-based biosensor that utilizes ion channels as the readout for binding of analytes to the biosensor.

In future work, we will construct a more robust biosensor by incorporating bis-gA channels into a BLM, where we can observe the activity of a single bis-gA channel and investigate in

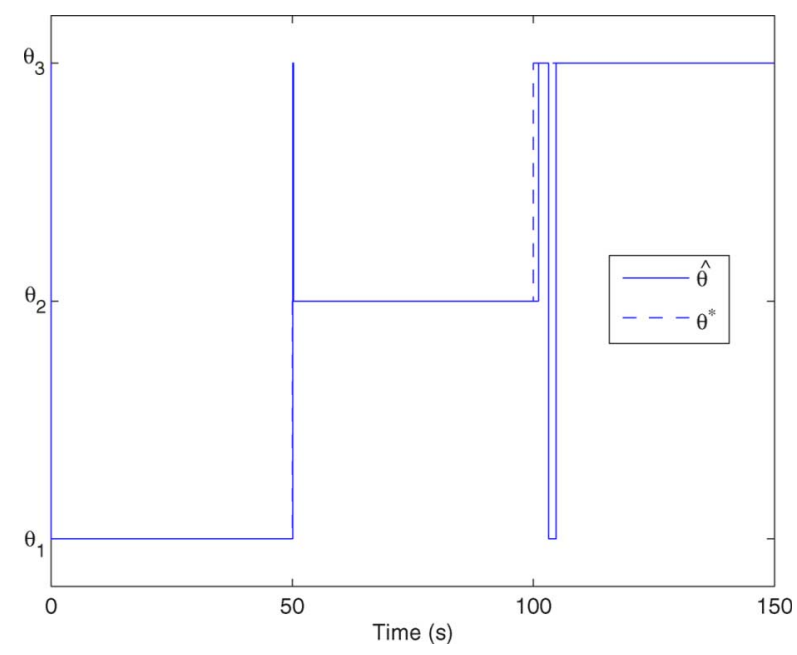

Fig. 14. Detection trace $\hat{\theta}$, generated using the likelihoods in Fig. 13, showing the most likely condition. The algorithm performed very well even when three different experimental conditions were involved.

more detail biological properties of the bis-gA channels. In particular, we will study the gating mechanism and whether coupling exists between neighboring channels.

\section{REFERENCES}

[1] B. A. Cornell, V. L. Braach-Maksvytis, L. G. King, P. D. Osman, B. Raguse, L. Wieczorek, and R. Pace, "A biosensor that uses ion channel switches," Nature, vol. 387, pp. 580-583, 1997.

[2] A. Anastasiadis and F. Separovic, "Solid-state NMR structural determination of components in an ion channel switch biosensor," Australian J. Chem., vol. 56, pp. 163-166, 2003.

[3] Y. Ephraim and N. Merhav, "Hidden Markov processes," IEEE Trans. Inf. Theory, vol. 48, pp. 1518-1569, Jun. 2002.

[4] I. L. di Maio, D. Carl, P. Langehanenberg, S. M. Valenzuela, A. R. Battle, S. al Khazally, M. Killingsworth, B. Kemper, G. von Bally, and D. K. Martin, "Structural properties of liposomes from digital holographic microscopy," in Proc. SPIE, Jan. 2006, vol. 6036, pp. 422-430.

[5] K. Akashi, H. Miyata, H. Itoh, and K. Kinosita, "Formation of giant liposomes promoted by divalent cations: Critical role of electrostatic repulsion," Biophys. J., vol. 74, pp. 2973-2982, Jun. 1998.

[6] K. Akashi, H. Miyata, H. Itoh, and K. Kinosita, "Preparation of giant liposomes in physiological conditions and their characterization under an optical microscope," Biophys. J., vol. 71, pp. 3242-3250, Dec. 1996.

[7] Y. Yamashita, M. Oka, T. Tanaka, and M. Yamazaki, "A new method for the preparation of giant liposomes in high salt concentrations and growth of protein microcrystals in them," Biochimica Et Biophysica Acta-Biomembranes, vol. 1561, pp. 129-134, Apr. 2002.

[8] R. Sauve and E. Bamberg, "l/f noise in black lipid membranes induced by ionic channels formed by chemically dimerized gramicidin A," $J$. Membrane Biol., vol. 43, pp. 317-333, Nov. 1978.

[9] R. Sauve and G. Szabo, "Interpretation of 1/f fluctuations in ion conducting membranes," J. Theoret. Biol., vol. 113, pp. 501-516, Apr. 1985.

[10] L. R. Rabiner, "A tutorial on hidden Markov models and selected applications in speech recognition," Proc. IEEE, vol. 77, pp. 257-285, 1989.

[11] A. P. Dempster, M. M. Laird, and D. B. Rubin, "Maximum likelihood estimation from incomplete data via the EM algorithm," J. Royal Stat. Soc. B, vol. 39, pp. 1-38, 1977.

[12] S. H. Chung, V. Krishnamurthy, and J. B. Moore, "Adaptive processing techniques based on hidden Markov models for characterizing very small channel currents buried in noise and deterministic interferences," Proc. Phil. Trans. Roy. Soc. Lond. B, vol. 334, pp. 357-384, 1991.

[13] V. Krishnamurthy and A. Logothetis, "Iterative and recursive estimators for hidden Markov errors-in-variables models," IEEE Trans. Signal Process., vol. 44, pp. 629-639, Mar. 1996.

[14] G. E. P. Box and G. M. Jenkins, Time Series Analysis_Forecasting and Control. San Francisco, CA: Holden-Day, 1976. 
[15] G. E. P. Box and D. A. Pierce, "Distribution of residual autocorrelations in autoregressive-integrated moving average time series models," J. Amer. Stat. Assoc., vol. 65, pp. 1509-1526, Dec. 1970.

[16] "Making lipid membranes even tougher," J. Mater. Res., 2007, in press.

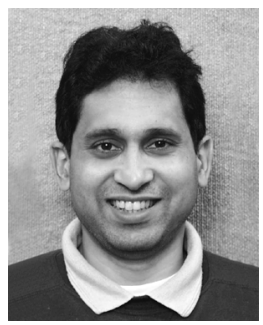

Vikram Krishnamurthy (S'90-M'91-SM'99F'05) was born in 1966. He received the B.S. degree from the University of Auckland, Auckland, New Zealand, in 1988 and the Ph.D. degree from the Australian National University, Canberra, Australia, in 1992.

Since 2002, he has been a Professor and Canada Research Chair at the Department of Electrical Engineering, University of British Columbia, Vancouver, BC, Canada. Prior to 2002, he was a Chaired Professor at the Department of Electrical and Electronic Engineering, University of Melbourne, Australia, where he also served as Deputy Head of department. He is co-editor of Biological Membrane Ion Channels-Dynamics Structure and Applications (Springer-Verlag, 2007), with $\mathrm{S}$. H. Chung and O. Andersen. His current research interests include stochastic modeling of biological ion channels, stochastic optimization and scheduling, and statistical signal processing.

Dr. Krishnamurthy has served as an Associate Editor for several journals including the IEEE TRANSACTIONS ON SIGNAL PROCESSING, the IEEE Transactions ON AEROSPACE AND ELECTRONIC SySTEMS, the IEEE TRANSACTIONS ON CIRCUITS AND SySTEMS B, and the IEEE TRANSACTIONS ON NANOBIOSCIENCE, and Systems and Control Letters.

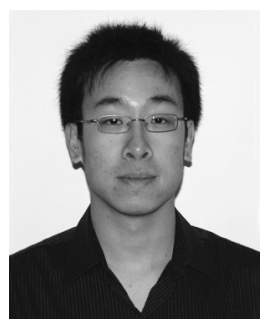

Kai Yiu Luk was born in 1982. He received the B.S. degree from the University of British Columbia (UBC), Vancouver, BC, Canada, in 2005. He is currently working towards the Master of Applied Science degree in electrical engineering at UBC.

His research interest include stochastic modeling of biological ion channels in the molecular level and macroscopic level.

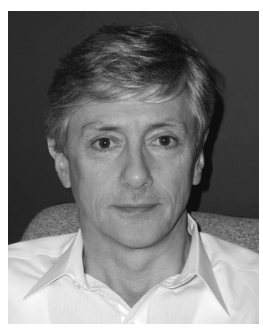

Bruce Cornell held a CSIRO Postdoctoral Fellowship at the University of London from 1975 to 1977 and returned to Australia to a senior scientific post with CSIRO in the field of biophysics. He was appointed the Director of the Cooperative Research Centre for Molecular Engineering (1992-1999) and founded the commercial consortium that became Ambri Ltd. for whom he worked as Chief Scientist (2000-2005). In 2005, he founded Surgical Diagnostics Ltd., where he is currently Director of Science and Technology. He is an elected member of the Australian Academy of Technological Sciences and Engineering and a member of a number of advisory groups to Australian Universities and an advisory to the Australian Government on the Medical Device Industry.
Jog Prasher photograph and biography not available at the time of publication.

Isabelle L. di Maio photograph and biography not available at the time of publication.

Hedayetul Islam photograph and biography not available at the time of publication.

Andrew R. Battle photograph and biography not available at the time of publication.

Stella M. Valenzuela photograph and biography not available at the time of publication.

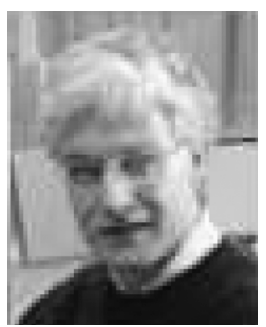

Donald K. Martin has formal training in optometry, biomedical engineering, and electrophysiology (postdoctoral). His postdoctoral training was at Sydney University by winning the inaugural Medical Foundation Postdoctoral Fellowship (1988-1991). He worked with Prof. I. Fatt at the University of California, Berkeley, and is currently an Associate Professor at the University of Technology, Sydney. $\mathrm{He}$ has edited a recent book on nanobiotechnology and the results of his research are reported in more than 70 publications in several international journals, including The Lancet, and have led to patents for a novel diagnostic system for diabetic retinopathy and a novel noninvasive drug delivery system. He initiated the formation of the Australian research network in nanobiotechnology called OzNano2Life (www.ambafrance-au.org/oznano2life). He is on the editorial board for two international journals in nanobiosciences. His contributions to science and technology are recognized formally in the Hansard Records of the NSW Parliament. His research interests are in nanobiotechnology, membrane transport, and ion channels.

Dr. Martin is an Executive Board Member of the Australian French Association for Science and Technology (AFAS NSW). He was invited to organize and chair panel discussions on nanobiotechnology for the Annual Conference of the Biotechnology Industry Organization (BIO) in 2003, 2004, and 2005. 